(C) 2012 IEEE. Reprinted, with permission, from S. Krone and G. Fettweis, Communications with 1-Bit Quantization and Oversampling at the Receiver: Benefiting from InterSymbol-Interference, in Proceedings of the 23rd IEEE International Symposium on Personal, Indoor and Mobile Radio Communications (PIMRC'12), Sydney, Australien, 9. 12. September 2012.

This material is posted here with permission of the IEEE. Such permission of the IEEE does not in any way imply IEEE endorsement of any of the products or services of Technical University Dresden. Internal or personal use of this material is permitted. However, permission to reprint/republish this material for advertising or promotional purposes or for creating new collective works for resale or redistribution must be obtained from the IEEE by writing to pubs-permissions@ieee.org. By choosing to view this document, you agree to all provisions of the copyright laws protecting it. 


\title{
Communications with 1-Bit Quantization and Oversampling at the Receiver: Benefiting from Inter-Symbol-Interference
}

\author{
Stefan Krone and Gerhard Fettweis \\ Vodafone Chair Mobile Communications Systems \\ Technische Universität Dresden, 01062 Dresden, Germany \\ E-mail: $\{$ stefan.krone, fettweis $\} @$ ifn.et.tu-dresden.de
}

\begin{abstract}
-1-bit analog-to-digital conversion is very attractive for low-complexity communications receivers. A major drawback is, however, the small spectral efficiency when sampling at symbol rate. This can be improved through oversampling by exploiting the signal distortion caused by the transmission channel. This paper analyzes the achievable data rate of band-limited communications channels that are subject to additive noise and intersymbol-interference with 1-bit quantization and oversampling at the receiver. It is shown that not only the channel noise but also the inter-symbol-interference can be exploited to benefit from oversampling.
\end{abstract}

\section{INTRODUCTION}

Multi-bit analog-to-digital converters (ADCs) at the receiver require an analog gain control to adjust the received signal amplitudes to the quantization grid of the ADCs [1]. This can be omitted when restricting to 1-bit quantization in order to reduce the complexity and the power dissipation of a communications receiver. The major drawback is, however, that the achievable data rate of a complex-valued signal transmission is then limited to 2 bits per channel use (bpcu), when the received signals are sampled at symbol rate. This can be improved through oversampling while maintaining the advantages of 1-bit quantization. This paper analyzes the prospects of 1bit quantization and oversampling for band-limited communications channels with inter-symbol-interference (ISI), which extends the authors' previous results published in [2] and [3].

Digital communications channels with coarse output quantization and oversampling have rarely been discussed, so far. This holds in particular true for an information-theoretic characterization of these kinds of channels. Known work has often considerd the overall statistics of the quantizer input signals and has not accounted for the fact that a received communications signal typically consists of a useful part and signal distortion caused by the transmission channel. Band-limited communications channels with 1-bit output quantization and oversampling have been analyzed in [4] for the special case without channel noise. It has been shown that 2-fold oversampling (with respect to the channel bandwidth) can improve the performance from $1 \mathrm{bpcu}$ to $1.072 \mathrm{bpcu}$, when Gaussian distributed channel inputs are used. With properly designed channel inputs, 2-fold oversampling can provide up to $2 \mathrm{bpcu}$. This has been derived in [5]. These results can be easily extended to complex-valued channels, which leads to twice the performan-

This work has been supported in part by the German Research Foundation in the framework of the Collaborative Research Center 912 "Highly Adaptive Energy-Efficient Computing" and by the European Social Fund in the framework of the Young Investigators Group "3D Chip-Stack Intraconnects". ce. The authors of [6] have derived the capacity of AWGN channels (not band-limited) with 1-bit output quantization and arbitrary integer oversampling for low signal-to-noise ratios (SNR). The achievable data rate of complex-valued AWGN channels with 1-bit quantization and oversampling has been analyzed for arbitrary SNRs in [2]. By considering conventional modulation schemes such as 16-QAM, it has been shown that the channel noise can be utilized as dither signal that increases the effective, quantization resolution and allows to detect higher-order modulation schemes even with 1-bit quantization. The analysis has been pursued in [3], by calculating the channel capacity and the capacity achieving transmit symbol constellations for arbitrary SNRs. This paper further extends the analysis by considering a more realistic system model that captures not only the AWGN of the channel but also the ISI caused by practical transmit and receive filters. The objectives of the paper are to provide a numerical framework and to show that the achievable data rate may not only benefit from the channel noise but also from the ISI. An exact calculation of the channel capacity, as considered in [3], is rather difficult. The analysis is therefore restricted to a numerical evaluation of the mutual information that can be achieved (at least) when equally probable 16-QAM symbols are transmitted. Even with this restriction it is possible to render the main effect, namely that random ISI can provide an additional performance gain, in particular at high SNR.

The paper is organized as follows: The system model is developed in Section II. Section III derives a mutual information lower bound that is considered to analyze the oversampling gain with additive channel noise, ISI and 1-bit quantization at the receiver. A numerical evaluation and a discussion of implications for optimal system designs are provided in Section IV. Section V concludes the paper with a short summary and outlines topics for further research.

\section{SySTEM MODEL}

Consider the equivalent baseband model of a communications system that transmits complex-valued data symbols $s[k]$ $=s_{\mathrm{I}}[k]+j \cdot s_{\mathrm{Q}}[k]$ over an AWGN channel with two digitalto-analog converters (DACs) at the transmitter and two 1-bit $\mathrm{ADCs}$ at the receiver (for the in-phase and quadrature-phase, respectively), as depicted in Fig. 1. The transmission channel is band-limited due to the filter characteristics of the transmitter and receiver, which are modeled by the impulse responses $g_{\mathrm{Tx}}(t)$ and $g_{\mathrm{Rx}}(t)$. The sampling rate of the transmitter DACs 


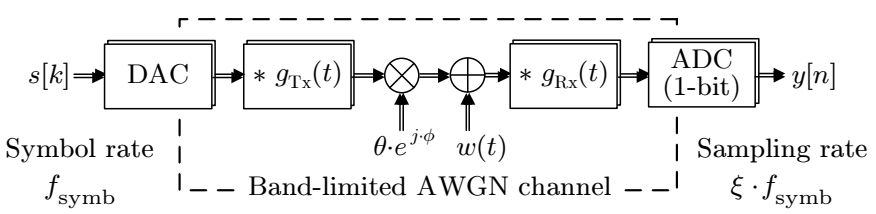

Fig. 1: Equivalent baseband model of a communications system that transmits complex-valued data symbols over a band-limited AWGN channel with 1-bit analog-to-digital conversion at the receiver.

is the same as the symbol rate $f_{\text {symb }}$, while the sampling rate of the 1-bit ADCs at the receiver is an integer multiple $(\xi)$ of $f_{\text {symb }}$. The DAC resolution is assumed to be sufficiently large to correctly represent the amplitudes of the transmitted data symbols. The DAC output waveforms are rectangular, which is modeled by the DAC impulse response $g_{\mathrm{DAC}}(t)=$ $\sqrt{f_{\text {symb }}} \cdot \operatorname{rect}\left(t \cdot f_{\text {symb }}-0.5\right)$, where $\operatorname{rect}(\cdot)$ denotes the rectangular function [7]. The factor $\sqrt{f_{\text {symb }}}$ scales the pulse shape to unit energy. $g_{\mathrm{DAC}}(t)$ is included in the overall transmitter impulse response $g_{\mathrm{Tx}}(t)$. Similarly, the impulse response $g_{\mathrm{Rx}}(t)$ of the receiver includes the sampling-and-hold behavior of the 1-bit ADCs, which is modeled as $g_{\mathrm{ADC}}(t)=$ $\sqrt{\xi \cdot f_{\text {symb }}} \cdot \operatorname{rect}\left(t \cdot \xi \cdot f_{\text {symb }}-0.5\right)$. The radio channel itself is assumed to be frequency flat, where $\theta$ denotes the overall channel attenuation, and $\phi$ is the channel phase. $\phi=0$, if the transmitter and receiver are perfectly synchronized in phase. The power spectral density of the AWGN $w(t)$ will be denoted as $\mathcal{N}_{0}$.

With these assumptions, a discrete time system model can be derived, which is shown in Fig. 2. It is considered at the ADC sampling rate, i.e, $\xi \cdot f_{\text {symb }}$. Thus, the quantized received samples write

$$
y[n]= \begin{cases}q_{1}+j \cdot q_{1} & \text { for } x_{\mathrm{I}}[n] \leq 0 ; x_{\mathrm{Q}}[n] \leq 0 \\ q_{1}+j \cdot q_{2} & \text { for } x_{\mathrm{I}}[n] \leq 0 ; x_{\mathrm{Q}}[n]>0 \\ q_{2}+j \cdot q_{1} & \text { for } x_{\mathrm{I}}[n]>0 ; x_{\mathrm{Q}}[n] \leq 0 \\ q_{2}+j \cdot q_{2} & \text { otherwise }\end{cases}
$$

where $q_{1}$ and $q_{2}$ denote the two 1-bit quantization levels of the in-phase and quadrature-phase, respectively, and the unquantized received samples are given as

$x[n]=x_{\mathrm{I}}[n]+j \cdot x_{\mathrm{Q}}[n]=$

$\sum_{n^{\prime \prime}=1}^{N_{\mathrm{ch}}} g_{\mathrm{ch}}\left[n^{\prime \prime}\right] \cdot s\left[\left\lfloor\left(n-n^{\prime \prime}+1\right) / \xi\right\rfloor\right]+\sum_{n^{\prime}=1}^{N_{\mathrm{Rx}}} g_{\mathrm{Rx}}\left[n^{\prime}\right] \cdot w\left[\frac{\xi_{w}}{\xi} \cdot n-n^{\prime}+1\right]$

with $\lfloor\cdot\rfloor$ denoting the smallest integer close to the value in brackets. $g_{\text {ch }}[n]$ is the discrete time impulse response of the overall transmission channel. It has a finite length $N_{\mathrm{ch}}$ and is for $1 \leq n \leq N_{\text {ch }}$ given as

$g_{\mathrm{ch}}[n]=\theta \cdot e^{j \cdot \phi} \cdot \int_{-\infty}^{\infty} g_{\mathrm{Rx}}\left(t^{\prime}\right) \cdot g_{\mathrm{Tx}}\left((n-1) /\left(\xi \cdot f_{\mathrm{symb}}\right)+\tau_{\mathrm{s}}-t^{\prime}\right) d t^{\prime}$,

where $\tau_{\mathrm{s}}$ denotes the sampling time offset between the transmitter and the receiver. The discrete time AWGN samples $w\left[n^{\prime}\right]$ and the discrete time receiver impulse response $g_{\mathrm{Rx}}\left[n^{\prime}\right]$ that shapes the AWGN are considered at $\xi_{w}$ times the ADC sampling rate to correctly account for the aliasing of the samp-

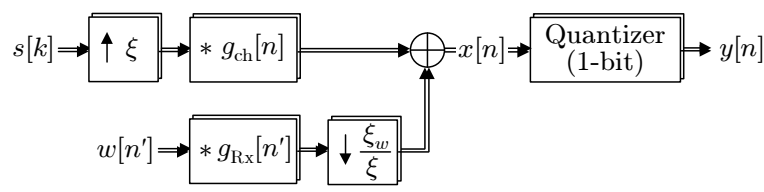

Fig. 2: Discrete time model of a communications system that transmits complex-valued data symbols over a band-limited AWGN channel with 1bit quantization and oversampling at the receiver. $\uparrow(\cdot)$ and $\downarrow(\cdot)$ denote (.)-fold up- and downsampling, respectively.

led noise. This assumes that the receiver bandwidth is strictly limited to $\pm \xi \cdot \xi_{w} \cdot f_{\text {symb }}$. Then, the AWGN samples follow from $w(t)$ as

$w\left[n^{\prime}\right]=$

$\int_{-\infty}^{\infty} \frac{\sin \left(\pi \cdot t^{\prime} \cdot \xi \cdot \xi_{w} \cdot f_{\mathrm{symb}}\right)}{\pi \cdot t^{\prime}} \cdot \frac{w\left(\left(n^{\prime}-1\right) /\left(\xi \cdot \xi_{w} \cdot f_{\mathrm{symb}}\right)+\tau_{\mathrm{s}}-t^{\prime}\right)}{\sqrt{\xi \cdot \xi_{w} \cdot f_{\mathrm{symb}}}} d t^{\prime}$

with zero mean and variance $\mathcal{N}_{0} \cdot g_{\mathrm{Rx}}\left[n^{\prime}\right]$ has length $N_{\mathrm{Rx}}$ and derives from the analog impulse response $g_{\mathrm{Rx}}(t)$ for $1 \leq n \leq$ $N_{\mathrm{Rx}}$ as

$g_{\mathrm{Rx}}\left[n^{\prime}\right]=g_{\mathrm{Rx}}\left(\left(n^{\prime}-1\right) /\left(\xi \cdot \xi_{w} \cdot f_{\mathrm{symb}}\right)\right) \cdot \sqrt{1 /\left(\xi \cdot \xi_{w} \cdot f_{\mathrm{symb}}\right)}$.

Assuming the transmitter and receiver impulse responses to have unit gain and considering i.i.d. zero-mean transmit symbols with variance $\sigma_{s}^{2}$, the signal-to-noise ratio (SNR) of the transmission channel follows as

$$
\gamma=\theta^{2} \cdot \sigma_{s}^{2} / \mathcal{N}_{0},
$$

which is the ratio of the average energy of a received symbol and the noise power spectral density. Note that any non-unit gain of the transmitter and receiver impulse responses can be considered as part of $\theta$.

When interested only in the received signal part that belongs to a particular symbol that has been transmitted at time instant $k$, the ISI caused by previously and later transmitted symbols can be separated from the useful signal parts to rewrite (2) as follows:

$$
\begin{aligned}
& x[n]=g_{\mathrm{ch}}[n] \cdot s[k]+\cdots \\
& \cdots \underbrace{\sum_{\substack{k^{\prime}=1 \\
k^{\prime} \neq k}}^{K} g_{\mathrm{ch}}\left[n-\xi \cdot\left(k^{\prime}-1\right)\right] \cdot s\left[k^{\prime}\right]}_{\text {ISI }}+\underbrace{\sum_{n^{\prime}=1}^{N_{\mathrm{Rx}}} g_{\mathrm{Rx}}\left[n^{\prime}\right] \cdot w\left[\frac{\xi_{w}}{\xi} \cdot n-n^{\prime}+1\right]}_{\text {noise }},
\end{aligned}
$$

which takes into account that $g_{\mathrm{ch}}[n]=0$ for $n<1$ and $n>N_{\mathrm{ch}}$. The ISI and the correlated noise can be regarded as random distortion which can be jointly exploited to improve the achievable data rate when the received samples $x[n]$ are coarsely quantized. This is considered and discussed in the next sections. The simplified case of an AWGN channel with rectangular pulse shapes, as considered in [2] and [3], is covered by (7), when $g_{\mathrm{ch}}[n]=\theta \cdot e^{j \cdot \phi} / \sqrt{\xi}$ for $1 \leq n \leq N_{\mathrm{ch}}$ with $N_{\mathrm{ch}}=\xi$ and $g_{\mathrm{Rx}}[n]=1 / \sqrt{\xi_{w}}$ for $1 \leq n \leq N_{\mathrm{Rx}}$ with $N_{\mathrm{Rx}}=\xi_{w} \cdot \xi$. Then, both the ISI and the correlation of the noise samples vanish. 

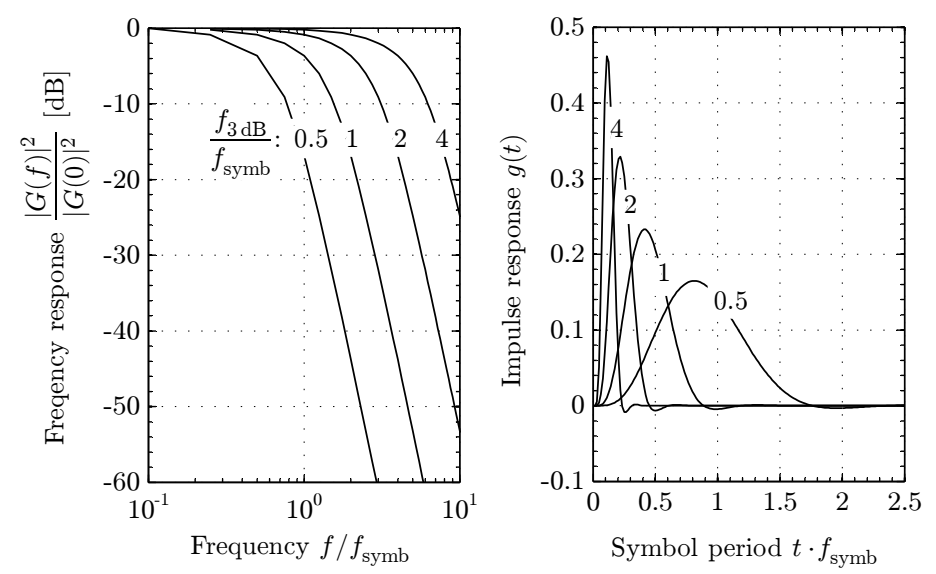

Fig. 3: Frequency and impulse responses of 5-th order Bessel low-pass filters for different $3-\mathrm{dB}$ cut-off frequencies (normalized to the symbol rate).

The analysis in this paper considers, however, practical transmit and receive filter characteristics that cause ISI. The filter characteristics are derived from the impulse responses of 5-th order Bessel low-pass filters [8], which are typical, e.g., for wideband communications transceivers [9]. The filter bandwidth at the transmitter equals the symbol rate $\left(f_{3 \mathrm{~dB}} / f_{\mathrm{symb}}=\right.$ 0.5 ), while the filter bandwidth at the receiver will be varied with the ADC sampling rate by adjusting the $3 \mathrm{~dB}$ cut-off frequency $f_{3 \mathrm{~dB}}$ accordingly. The latter is to study the impact of different receiver bandwidths and sampling rates. Fig. 3 shows the frequency and impulse responses of the considered filters. The Bessel filter impulse responses are convolved with the rectangular impulse responses of the transmitter DACs and the receiver ADCs, as stated before, which yields $g_{\mathrm{Tx}}(t)$ and $g_{\mathrm{Rx}}(t)$. The particular type of the band-limiting filters is of less importance for the effects discussed in this paper, as long as the band-limitation leads to ISI. Also, note that matched receive filtering, which would maximize the SNR of the received symbols at the quantizer input is not considered here, since the results discussed in [2] and [3] have already indicated that a high SNR is not necessarily optimal to maximize the achievable data rate with 1-bit quantization and oversampling at the receiver.

The impulse responses $g_{\mathrm{ch}}[n]$ and $g_{\mathrm{Rx}}[n]$ for the discrete time representation of the system model are computed from the Bessel filters and the rectangular impulse responses of the transmitter DACs and receiver ADCs by approximating each of the continuous-time impulse responses $g_{\mathrm{Tx}}(t)$ and $g_{\mathrm{Rx}}(t)$ with a quasi-analog sampling rate that is 64 times the symbol rate. The length of $g_{\mathrm{ch}}[n]$ and $g_{\mathrm{Rx}}[n]$ has been terminated to 4 symbol periods, i.e., $N_{\mathrm{ch}}=4 \cdot \xi$ and $N_{\mathrm{Rx}}=4 \cdot \xi_{w} \cdot \xi$. The noise oversampling ratio is in all considered cases $\xi_{w}=2$.

\section{Mutual Information Lower Bound}

The system performance is evaluated in terms of the achievable data rate that follows from the mutual information of the transmission channel. To compute the mutual information it is necessary to reformulate the system model such that the channel with memory is translated into an equivalent memoryless channel. This is possible by considering transmit and receive vectors of length $K$ and $N$, respectively, which span over multiple symbols. The mutual information per transmit symbol is then (strictly) given as the mutual information between the transmit and receive vectors by letting $K$ and $N$ tend to infinity [10]. Thus, by stacking the transmitted symbols and the received samples in vectors of length $K$ and $N=\xi \cdot K+N_{\mathrm{ch}}-1$, and assuming a discrete transmit symbol alphabet with cardinality $L$, e.g., $L$-QAM, the mutual information per transmit symbol can be written as

where

$$
\mathrm{I}(S ; Y)=\lim _{K \rightarrow \infty} \frac{1}{K} \cdot \mathrm{I}(\boldsymbol{S} ; \boldsymbol{Y})
$$

$$
\begin{array}{r}
\mathrm{I}(\boldsymbol{S} ; \boldsymbol{Y})=\sum_{\alpha=1}^{4^{N}} \sum_{\kappa=1}^{L^{K}} \operatorname{Pr}\left(\boldsymbol{y}=\mathbf{y}_{\alpha} \mid \boldsymbol{s}=\mathbf{s}_{\kappa}\right) \cdot \operatorname{Pr}\left(\boldsymbol{s}=\mathbf{s}_{k}\right) \\
\cdot \log _{2}\left(\frac{\operatorname{Pr}\left(\boldsymbol{y}=\mathbf{y}_{\alpha} \mid \boldsymbol{s}=\mathbf{s}_{\kappa}\right)}{\sum_{\kappa^{\prime}=1}^{L^{K}} \operatorname{Pr}\left(\boldsymbol{y}=\mathbf{y}_{\alpha} \mid \boldsymbol{s}=\mathbf{s}_{\kappa^{\prime}}\right) \cdot \operatorname{Pr}\left(\boldsymbol{s}=\mathbf{s}_{\kappa^{\prime}}\right)}\right)
\end{array}
$$

with $\operatorname{Pr}\left(s=\mathbf{s}_{\kappa}\right)$ denoting the probabilities of the transmit symbol vectors. The transition probabilities $\operatorname{Pr}\left(\boldsymbol{y}=\mathbf{y}_{\alpha} \mid \boldsymbol{s}=\mathbf{s}_{\kappa}\right)$ can be derived as $N$-dimensional complex integrals over an $N$-variate complex Gaussian probability density function (PDF) each, where the integral limits correspond to the 1-bit quantization thresholds.

For transmit and receive vectors of infinite length it is rather impossible to compute the mutual information numerically. To still allow for a numerical performance analysis (but also to adjust the data rate in a practical system), a reasonable solution is to consider a lower bound of the mutual information which treats the ISI between different received symbols as random distortion and can be computed numerically. This is considered in the following.

\section{A. Calculation of the lower bound}

The considered lower bound is based on the assumption that the receiver detects each transmitted symbol individually without accounting for a dependency between the received samples of different symbols. To model this, a sequence of $K$ sub-vectors $\underline{\boldsymbol{y}}_{k}$ is extracted out of the overall receive vector $\boldsymbol{y}$, where each $\underline{\boldsymbol{y}}_{k}$ contains the $\xi$ received samples of one symbol period. That is,

$$
\left[\underline{\boldsymbol{y}}_{k}\right]_{n}=y\left[\xi \cdot(k-1)+n+n_{\text {delay }}\right],
$$

where $1 \leq n \leq \xi$, and $n_{\text {delay }}$ is a sample delay that defines the start of the detection interval within the received waveform of each symbol. A reasonable way to define an optimal sample delay $n_{\text {delay }}$ is to maximize the overall sample energy integrated over the symbol detection period, i.e.,

$$
\begin{aligned}
n_{\text {delay }} & =\arg \max _{n} \sum_{n^{\prime}=0}^{\xi-1}\left|g_{\mathrm{ch}}\left[n+n^{\prime}\right]\right|^{2}-1 \\
& =\arg \max _{n} \sum_{n^{\prime}=0}^{\xi-1}\left|\int_{\left(n+n^{\prime}-1\right) /\left(\xi \cdot f_{\mathrm{symb}}\right)}^{\left(n+n^{\prime}\right) /\left(\xi \cdot f_{\mathrm{symb}}\right)} \sqrt{\xi \cdot f_{\mathrm{symb}}} \cdot \breve{g}_{\mathrm{ch}}(t) d t\right|^{2}-1,
\end{aligned}
$$


where $n \in\left\{1,2, \ldots, N_{\mathrm{ch}}-\xi+1\right\}$, and $\breve{g}_{\mathrm{ch}}(t)$ denotes the pulse shape before the integration and sampling of the ADCs at the receiver. This is illustrated for $\theta=1$ and $\phi=0$ in Fig. 4. Note that the definition of $n_{\text {delay }}$ in (11) leads always to the same integrated sample energy over a symbol period, regardless of the particular OSR at the receiver to allow for a reasonable comparison of different sampling rates.

To calculate the mutual information lower bound, let $\underline{y}$ denote a generalized vector of length $\xi$ that can assume all $\overline{4}$ possible received sample combinations $\underline{\mathbf{y}}_{\alpha}$ of $\underline{\boldsymbol{y}}_{k}$. Furthermore, consider a DMC which has the scalar inputs $s$ and the output vectors $\boldsymbol{y}$. For equally probable i.i.d. transmit symbols, it can then be shown that the mutual information $\mathrm{I}(S ; \underline{\boldsymbol{Y}})$ of this DMC is a lower bound to the mutual information per transmitted symbol of the true channel. That is,

$$
\begin{aligned}
\frac{1}{K} \cdot \mathrm{I}(\boldsymbol{S} ; \boldsymbol{Y}) \geq \mathrm{I}(S ; \underline{\boldsymbol{Y}})= & \sum_{\alpha=1}^{4^{\xi}} \sum_{l=1}^{L} \frac{1}{L} \cdot \overline{\operatorname{Pr}}_{K}\left(\underline{\boldsymbol{y}}=\underline{\mathbf{y}}_{\alpha} \mid s=\mathrm{s}_{l}\right) \quad(12) \\
& \cdot \log _{2}\left(\frac{\overline{\operatorname{Pr}}_{K}\left(\underline{\boldsymbol{y}}=\underline{\mathbf{y}}_{\alpha} \mid s=\mathrm{s}_{l}\right)}{\sum_{l^{\prime}=1}^{L} \frac{1}{L} \cdot \overline{\operatorname{Pr}}_{K}\left(\underline{\boldsymbol{y}}=\underline{\mathbf{y}}_{\alpha} \mid s=\mathrm{s}_{l^{\prime}}\right)}\right) .
\end{aligned}
$$

which can be derived from the chain rule of mutual information [10]. The marginal transition probabilities in (12) follow for $k \rightarrow \infty$ as

$$
\begin{aligned}
\lim _{K \rightarrow \infty} \overline{\operatorname{Pr}}_{K}\left(\underline{\boldsymbol{y}}=\underline{\mathbf{y}}_{\alpha} \mid s=\mathbf{s}_{l}\right)= & \lim _{K \rightarrow \infty} \frac{1}{K} \cdot \sum_{k=1}^{K} \operatorname{Pr}\left(\underline{\boldsymbol{y}}_{k}=\underline{\mathbf{y}}_{\alpha} \mid[\boldsymbol{s}]_{k}=\mathbf{s}_{l}\right) \\
& \left.=\int_{\left[\hat{\boldsymbol{\chi}}_{\alpha}\right]_{1}} \ldots \int_{\left[\tilde{\boldsymbol{\chi}}_{\alpha}\right]_{1}} \ldots \mathrm{p}_{\underline{\underline{\boldsymbol{x}}}} \mid s\right]_{\xi}\left(\underline{\boldsymbol{x}} \mid \mathrm{s}_{l}\right) d[\underline{\boldsymbol{x}}]_{1} \ldots d[\underline{\boldsymbol{x}}]_{\xi}
\end{aligned}
$$

where the integrals are defined in the complex domain, and the vectors $\hat{\chi}_{\alpha}$ and $\check{\chi}_{\alpha}$ contain the 1-bit quantization thresholds that correspond to the quantization levels in the output vector $\underline{\mathbf{y}}_{\alpha}$. The conditional PDF $\underline{\mathrm{p}}_{\underline{\boldsymbol{x}} \mid s}\left(\underline{\boldsymbol{x}} \mid \mathrm{s}_{l}\right)$ is a weighted sum of the multi-variate complex Gaussian PDFs that result for each possible transmit symbol sequence $s$ where $[s]_{k}=\mathrm{s}_{l}$. This conditional PDF can be derived as follows:

Let $\underline{\boldsymbol{x}}$ denote the column vector of length $\xi$, which contains the received samples of one symbol period before the quantization. Using a matrix notation for the considered system model, $\underline{\boldsymbol{x}}$ can be written as

$$
\underline{\boldsymbol{x}}=\boldsymbol{G}_{\mathrm{ch}} \cdot \mathfrak{U} \cdot \underline{\boldsymbol{s}}+\mathfrak{D} \cdot \boldsymbol{G}_{\mathrm{Rx}} \cdot \underline{\boldsymbol{w}},
$$

where $\underline{s}$ is a vector of length $\left(N_{\mathrm{ch}}+\xi-1\right) / \xi$ which contains the transmitted symbols that affect the received samples in $\underline{x}$. The length assumes that $N_{\mathrm{ch}}-1$ is an integer multiple of $\xi$. If this is not the case, the considered channel impulse response can be zero-padded for the appropriate length. The intended symbol $\mathrm{s}_{l}$ is the $\left(\left\lceil n_{\text {delay }} / \xi\right\rceil+1\right)$-th element of the vector. The vector can have $L^{\left(N_{\mathrm{ch}}-1\right) / \xi}$ different realizations $\mathbf{s}_{i}$ for a given symbol $\mathrm{s}_{l}$. The matrix $\mathfrak{U}$ in (14) is an upsampling matrix of size $\left(N_{\mathrm{ch}}+\xi-1\right) \times\left(\left(N_{\mathrm{ch}}+\xi-1\right) / \xi\right)$, which accounts also for the sample delay at the receiver and has elements

$[\mathfrak{U}]_{n, n^{\prime}}= \begin{cases}1 & \text { for } n=\xi \cdot n^{\prime}-\left(\xi-1-\left(n_{\text {delay }}-1\right) \bmod \xi\right) \\ 0 & \text { otherwise. }\end{cases}$ The matrix $\boldsymbol{G}_{\mathrm{ch}}$ is a convolution matrix of the channel impulse response. It has size $\xi \times\left(N_{\mathrm{ch}}+\xi-1\right)$ and elements

$$
\left[\boldsymbol{G}_{\mathrm{ch}}\right]_{n, n^{\prime}}= \begin{cases}g_{\mathrm{ch}}\left[n^{\prime}-n+1\right] & \text { for } 1 \leq n^{\prime}-n+1 \leq N_{\mathrm{ch}} \\ 0 & \text { otherwise. }\end{cases}
$$

Similarly, $\boldsymbol{G}_{\mathrm{Rx}}$ denotes the convolution matrix of the receiver impulse response, which has size $\left(\xi_{w} \cdot \xi\right) \times\left(N_{\mathrm{Rx}}+\xi \cdot \xi_{w}-1\right)$ and elements

$\left[\boldsymbol{G}_{\mathrm{Rx}}\right]_{n, n^{\prime}}= \begin{cases}g_{\mathrm{Rx}}\left[n^{\prime}-n+1\right] & \text { for } 1 \leq n^{\prime}-n+1 \leq N_{\mathrm{Rx}} \\ 0 & \text { otherwise. }\end{cases}$

For preciseness, it should be noted that the Toeplitz structure [11] of the convolution matrices $\boldsymbol{G}_{\mathrm{ch}}$ and $\boldsymbol{G}_{\mathrm{Rx}}$ does not exactly hold for the very first samples of a transmitted symbol sequence. This is ignored here, since the sequence of trans-

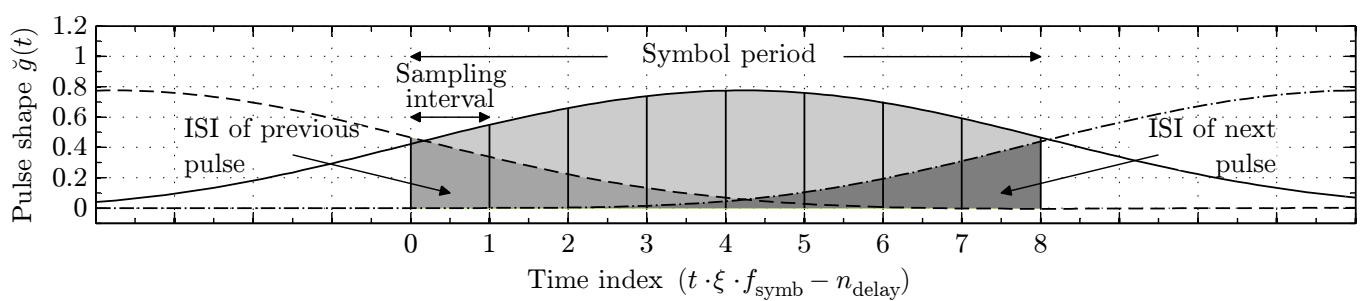

Fig. 4: Delay-optimized sampling intervals for the received pulse shapes with 5-th order Bessel low-pass filters at the transmitter and receiver. The depicted example considers Bessel filters with $f_{3 \mathrm{~dB}} / f_{\mathrm{symb}}=0.5$ at the transmitter, $f_{3 \mathrm{~dB}} / f_{\text {symb }}=4$ at the receiver and 8 -fold oversampling.
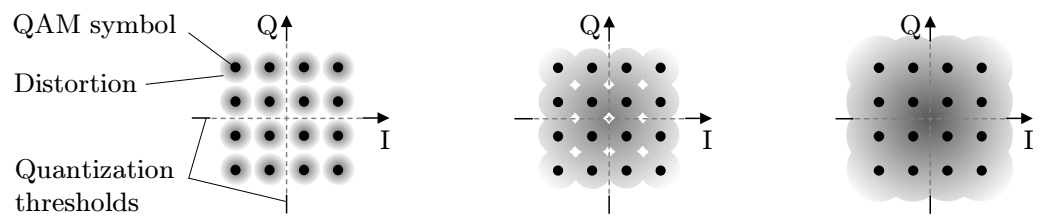

Fig. 5: Received 16-QAM constellation diagrams (with different levels of distortion) and 1-bit quantization thresholds.

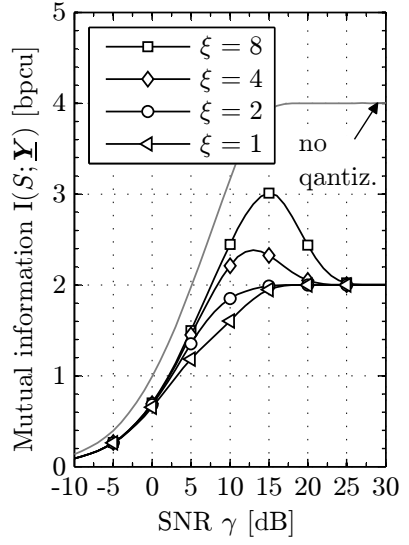

Fig. 6: Mutual information for 16QAM symbol transmission over AWGN channels with 1-bit output quantization and $\xi$-fold oversampling (without band-limitation and ISI). 

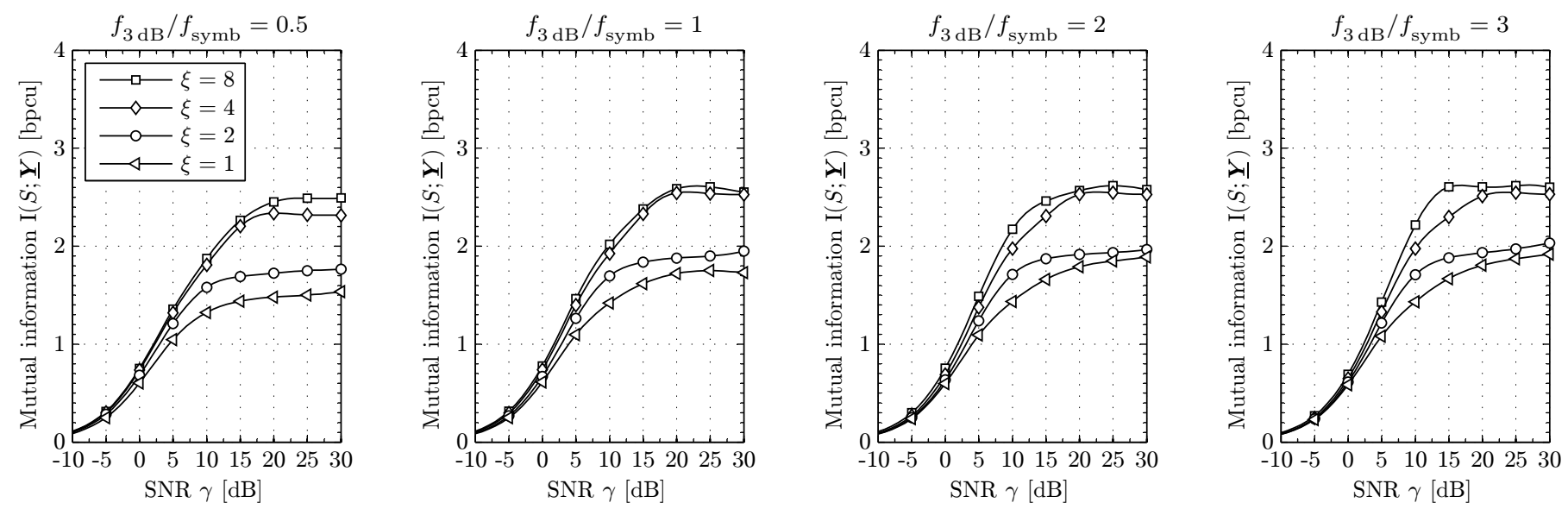

Fig. 7: Mutual information lower bounds for 16-QAM symbol transmission over band-limited AWGN channels with 1-bit output quantization and $\xi$-fold oversampling. The band-limitation and ISI result from 5-th order Bessel filters at the transmitter with $f_{3 \mathrm{~dB}} / f_{\text {symb }}=0.5$ and the same filter type at the receiver, considering different ratios $f_{3 \mathrm{~dB}} / f_{\mathrm{symb}}$.

mitted symbols is typically much longer than the filter length, such that the effect of the approximation is negligible. The noise vector $\underline{\boldsymbol{w}}$ is a column vector of length $N_{\mathrm{Rx}}+\xi_{w} \cdot \xi-1$. The elements of $\underline{\boldsymbol{w}}$ are complex-valued AWGN samples with zero mean and variance $\mathcal{N}_{0}$. Finally, $\mathfrak{D}$ denotes a downsampling matrix of size $\xi \times\left(\xi_{w} \cdot \xi\right)$ with elements

$$
[\mathfrak{D}]_{n, n^{\prime}}= \begin{cases}1 & \text { for } n=\xi_{w} / \xi \cdot\left(n^{\prime}-1\right)+1 \\ 0 & \text { otherwise. }\end{cases}
$$

With this matrix notation, $\underline{\mathrm{p}}_{\underline{x}} \mid s\left(\underline{\boldsymbol{x}} \mid \mathrm{s}_{l}\right)$ can be stated in closedform as given in (19), where $\operatorname{det}(\cdot)$ denotes the determinant of a matrix [11]. Using (19), the marginal transition probabilities in (13) can be computed explicitly by solving the multi-dimensional integrals. An alternative solution is to obtain the marginal transition probabilities from Monte-Carlo simulations. That is, the transition probabilities are calculated as the relative frequencies of the simulated received vectors $\underline{\mathbf{y}}_{\alpha}$ given the transmit symbols $s_{l}$. These transition probabilities become more and more exact the more transmit symbols are simulated. This solution has been used for the numerical analysis of the mutual information lower bound in Section IV.

\section{B. Possible extensions}

The considered lower bound can be easily extended to observing more than $\xi$ samples per transmit symbol. That is, the samples in the received vector $\boldsymbol{y}$ can in principle also span over multiple symbol periods. This can improve the lower bound to some extend. The improvement is, however, rather small for the considered examples, since the main part of the received symbol energy is contained within one symbol period. Another valuable extension would be a generalization of the mutual information lower bound to non-integer oversampling. For an analysis of the principle behavior, as considered in this paper, it is, however, sufficient to restrict to integer OSRs.

\section{NUMERICAL EVALUATION AND IMPLICATIONS}

Similar as in [2], 16-QAM transmit symbols are considered. That is, the cardinality of the modulation scheme is larger than the quantization resolution at the receiver, but the oversampling is expected to increase the effective quantization resolution by exploiting the signal distortions. The channel phase is assumed to be perfectly equalized such that $\phi=0$.

The mutual information that results for AWGN channels without band-limitation and ISI, as considered in [2], is shown in Fig. 6. In this case, $\mathrm{I}(S ; \underline{\boldsymbol{Y}})$ is not only a lower bound but true the mutual information of the channel. It can be seen that oversampling can improve the mutual information per transmitted symbol to more than 2 bpcu by exploiting the channel noise. The reason is that multiple samples of the same received symbol may be mapped to different quantization levels due to the noise, as illustrated in Fig. 5. This effect depends on the distance of a received symbol to the quantization thresholds, which in turn allows to resolve 16-QAM symbols from 1bit quantized samples. With oversampling, I $(S ; \underline{\boldsymbol{Y}})$ attains its maximum at an optimal SNR below infinity. This can be referred to as stochastic resonance. Since the oversampling gain relies on the channel noise, $\mathrm{I}(S ; \underline{\boldsymbol{Y}})$ drops back to $2 \mathrm{bpcu}$ at high SNR.

This can be mitigated by reducing the transmit signal power or by introducing artificial signal distortion such as ISI. For band-limited channels, as discussed in this paper, the overall distortion is composed ISI and (correlated) channel noise, as indicated in (7). Fig. 7 shows the respective mutual information lower bound, which has been computed with MonteCarlo simulation results of $K=10^{8}$ randomly transmitted 16-QAM symbols. Multiple effects can be observed: Without and with little oversampling $(\xi \leq 2)$, the mutual information lower bound degrades as compared to the case without ISI.

$$
\mathrm{p}_{\underline{\boldsymbol{x}} \mid s}\left(\underline{\boldsymbol{x}} \mid \mathrm{s}_{l}\right)=\sum_{i=1}^{L^{\left(N_{\mathrm{ch}}+\xi-1\right) / \xi}} \frac{1}{L^{\left(N_{\mathrm{ch}}+\xi-1\right) / \xi}} \cdot \frac{\exp \left(\frac{1}{\xi \cdot \sigma_{w}^{2}} \cdot\left(\underline{\boldsymbol{x}}-\boldsymbol{G}_{\mathrm{ch}} \cdot \mathfrak{U} \cdot \underline{\mathbf{s}}_{i}\right)^{\mathrm{H}} \cdot\left(\boldsymbol{G}_{\mathrm{Rx}}^{\mathrm{H}} \cdot \mathfrak{D}^{\mathrm{H}} \cdot \mathfrak{D} \cdot \boldsymbol{G}_{\mathrm{Rx}}\right)^{-1} \cdot\left(\underline{\boldsymbol{x}}-\boldsymbol{G}_{\mathrm{ch}} \cdot \mathfrak{U} \cdot \underline{\mathbf{s}}_{i}\right)\right)}{(2 \cdot \pi)^{\xi} \cdot \xi \cdot \sigma_{w}^{2} \cdot \operatorname{det}\left(\boldsymbol{G}_{\mathrm{Rx}}^{\mathrm{H}} \cdot \mathfrak{D}^{\mathrm{H}} \cdot \mathfrak{D} \cdot \boldsymbol{G}_{\mathrm{Rx}}\right)}
$$


Even at high SNR, the achievable rate stays below 2 bpcu. This is mainly due to the distortion of the ISI, but also due to the time dispersion that spreads part of the symbol energy out of the detection interval. This effect is more pronounced the smaller the receive filter bandwidth is. However, larger oversampling ratios can improve the performance. At medium SNR, the performance with oversampling can again exceed 2 bpcu. The larger the receiver bandwidth is, the higher is the mutual information that can be achieved with stochastic resonance at an optimal SNR. This is due to the fact that the correlation of the noise samples decreases with a larger receiver bandwidth, which then improves the performance with oversampling.

The most important observation is, however, that the mutual information with oversampling can stay above 2 bpcu even at high SNR due to the ISI. This effect results from the randomness of the ISI, which improves the mutual information similarly as the channel noise. Beside the different statistics, the major difference is that the distortion due to the ISI does not vanish at high SNR. Note, however, that the mutual information stays above 2 bpcu only if the ISI is sufficiently large to affect the quantization. That is, if the maximum amplitude of the ISI would be less than the amplitude of the received samples without ISI, none of the quantized received samples would be affected by the ISI at high SNR, and the mutual information would return to $2 \mathrm{bpcu}$. This is not the case with the considered Bessel filters, but it could occur with different filter characteristics and a larger transmitter bandwidth. The rather small improvement of $\mathrm{I}(S ; \underline{\boldsymbol{Y}})$ when the OSR is increased from $\xi=4$ to $\xi=8$ can be traced back to the fact that the ISI within a symbol interval stems only from the previous and the next symbol (see Figure 4). This confines the amplitude space of the random ISI and prevents larger improvements at high OSRs for a 16-QAM symbol alphabet and the considered filter characteristics. If the overall channel impulse response would have a larger ripple before and/or after the symbol detection interval, more symbols would contribute to the ISI. This would increase the amplitude space of the ISI and could further improve the oversampling gain at high OSRs.

The general conclusion to be drawn from the above observations is that random ISI can be exploited similarly as AWGN to improve the system performance with 1-bit quantization and oversampling at the receiver. Note that the mutual information shown in Fig. 7 is still a lower bound. It indicates a data rate that can be achieved at least. The oversampling gain depends on the ISI but also on the filtered AWGN, both of which jointly affect the received samples of each symbol. It can be conjectured, that there is an optimal trade-off between the degradation caused by the overall distortion and its benefit in terms of an increased effective quantization resolution. As both effects depend on the transmit and receive filter characteristics, an optimal trade-off will require optimal filter designs, where the transmit filter design may be constrained in practice by spectral mask requirements. Optimal receive filters will not only optimize the ISI but also the bandwidth of the additive noise. Regarding an optimization in terms of the ISI, there will be a trade-off between the symbol energy that is kept in the detection interval and the part that is spread out to distort other symbols optimally. As the ISI does not only depend on the filter characteristics but also on the transmit symbol alphabet, a joint optimization is eventually required to maximize the system performance. The detection delay $n_{\text {delay }}$ can be considered as another degree of freedom for the optimization.

\section{CONCLUSIONS}

This paper has considered band-limited communications systems with 1-bit quantization and oversampling at the receiver. A numerical analysis based on a (proven) lower bound of the mutual information has been carried out to show that the system performance can benefit not only from the channel noise but also from ISI. That is, ISI can also improve the mutual information per transmitted symbol with oversampling to more that $2 \mathrm{bpcu}$, in particular at high SNR.

This motivates the design of optimal transmit and receive filters to maximize the achievable data rate of communications systems with 1-bit quantization and oversampling at the receiver. The optimization is in the focus of further research. An example application is the design of low-complexity transceivers for energy-efficient wireless wide-band communication links that are needed in next generation high-performance computers [12]. It is expected that the maximum mutual information that has been calculated in [3] for AWGN channels without ISI, can be exceeded with the design of dedicated filter characteristics and optimal channel inputs. Another open research topic is to extend the results of this paper to multi-bit quantization and to non-integer oversampling ratios.

\section{REFERENCES}

[1] S. Krone and G. Fettweis, "Optimal Gain Control for Single-Carrier Communications with Uniform Quantization at the Receiver," Proceedings of the IEEE International Conference on Acoustics, Speech and Signal Processing (ICASSP'10), 2010.

[2] _ "Achievable Rate with 1-Bit Quantization and Oversampling at the Receiver," IEEE Communication Theory Workshop (CTW'10), 2010.

[3] _ - "Capacity of Communications Channels with 1-Bit Quantization and Oversampling at the Receivers," Proceedings of the IEEE Sarnoff Symposium (Sarnoff'12), 2012.

[4] E. N. Gilbert, "Increased Information Rate by Oversampling," IEEE Transactions on Information Theory, vol. 39, no. 6, 1993.

[5] S. Shamai, "Information Rates by Oversampling the Sign of a Bandlimited Process," IEEE Trans. Information Theory, vol. 40, no. 4, 1994.

[6] T. Koch and A. Lapidoth, "Increased Capacity per Unit-Cost by Oversampling," IEEE Convention of Electrical and Electronics Engineers in Israel (IEEEI'10), 2010.

[7] S. Haykin and B. van Veen, Signals and Systems. John Wiley \& Sons, Inc., 1998.

[8] G. Bianchi and R. Sorrentino, Electronic Filter Simulation and Design. McGraw- Hill, Inc., 2007.

[9] M. Varonen, M. Kaltiokallio, V. Saari, O. Viitala, M. Kärkkäinen, S. Lindfors, J. Ryynänen, and K. A. I. Halonen, "A $60-\mathrm{GHz}$ CMOS Receiver With an On-Chip ADC," Proccedings of the IEEE RFIC Symposium, 2009

[10] R. G. Gallager, Information Theory and Reliable Commuunication. John Wiley \& Sons, Inc., 1968.

[11] I. N. Bronstein, K. A. Semendjajew, G. Musiol, and H. Mühlig, Handbook of Mathematics. Springer-Verlag, 2007.

[12] Collaborative Reserach Center "Highly Adaptive Energy-Efficient Computing (HAEC)", [Online]. Webpage: tu-dresden.de/sfb912, 2012. 